\title{
Adolescentes heridos por arma de fuego y sus factores biosocioeconómicos relacionados
}

Adolescents injured by firearm and their related biosocioeconomic factors

\section{Oscar Ponce Barahona*, Oscar Ponce Quezada**}

\section{RESUMEN}

Antecedentes: En los últimos años las heridas por arma de fuego (HPAF) se han incrementado a nivel mundial; son la segunda causa de muerte traumática en adolescentes en los Estados Unidos. En Honduras se ha incrementado esta patología probablemente debido a factores de diversa índole tales como: desintegración familiar, desempleo, incorporación temprana a grupos delictivos, experiencias con drogas, difusión no controlada de actos violentos a través de medios de comunicación masivos y facilidad para adquisición de armas. Objetivo: Determinar los factores biológicos, sociales y económicos relacionados con HPAF en pacientes adolescentes atendidos en la Emergencia de Pediatría del Hospital Mario Catarino Rivas (HMCR), en el período de julio 2015 -junio 2017. Pacientes y métodos: estudio descriptivo, observacional, prospectivo, con muestreo no probabilístico por conveniencia. Resultados: la prevalencia fue de $4.1 \%$. Entre los adolescentes con HPAF los factores biosocioeconómicos identificados a deserción escolar fueron: desintegración familiar, emancipación, pobreza, dependencia económica familiar, asociación a grupos delictivos, uso de alcohol y tabaco. Conclusiones: En este estudio predomino el sexo masculino y el grupo etario mas afectado fueron los adolescentes. Una gran parte residían en San Pedro Sula y también hubo relación con el sitio geográfico del trauma el cual fue el mismo. Cabe señalar que no predominaron las complicaciones médicas, pero el principal móvil del trauma fueron los asaltos.

\footnotetext{
*Médico Residente 3 Año de Posgrado de Pediatría UNAH-VS

** Médico Pediatra IHSS

Dirigir correspondecia a: oponce_1987@hotmail.com

Recibido: 10 de septiembre 2017 Aprobado: 10 de enero 2018
}

PALABRAS CLAVE

Heridas de bala, adolescente, arma de fuego.

\section{ABSTRACT \\ Background: In recent years, gunshot} wounds (HPAF) have increased worldwide; they are the second cause of traumatic death in adolescents in the United States. In Honduras this pathology has increased probably due to factors of various kinds such as: family disintegration, unemployment, early incorporation to criminal groups, drug experiences, uncontrolled dissemination of violent acts through mass media and ease of acquisition of weapons. Objective: To determine the biological, social and economic factors related to HPAF in adolescent patients treated in the Pediatric Emergency of the Hospital Mario Catarino Rivas, in the period of July 2015 -June 2017. Patients and methods: descriptive, observational, prospective study, with non-probabilistic sampling for convenience. Results: the prevalence was $4.1 \%$. Among adolescents with HPAF, the biosocioeconomic factors identified as dropouts were: family disintegration, emancipation, poverty, family economic dependence, association with criminal groups, alcohol and tobacco use. Conclusions: In this study, the male sex predominated and adolescents were the most affected age group. A large part resided in San Pedro Sula and there was also a relationship with the geographical location of the trauma, which was the same. It should be noted that medical complications did not predominate, but the main motive of the trauma was assaults.

\section{KEY WORDS}

Wounds, gunshot, adolescent, firearm. 


\section{INTRODUCCIÓN}

Según autores de Medicina Legal, las HPAF son: "aquellos efectos lesivos que producen sobre el organismo los disparos realizados con armas cargadas con diversos tipos de pólvora u otros explosivos", definición que excluye a las heridas por armas de aire comprimido.(1) Según las estadísticas a nivel mundial, las HPAF tienen una importancia creciente en la salud de la población pediátrica, principalmente en los países en vías de desarroIlo. ${ }^{(2)}$ En los últimos años las lesiones por proyectil de arma de fuego se han incrementado en todo el mundo; constituyendo actualmente un problema de salud pública. Son la segunda causa de muerte traumática en los adolescentes en los Estados Unidos ${ }^{(2)}$ en México y Venezuela se ha incrementado la frecuencia de estas lesiones aunque no se dispone de una estadística real. ${ }^{(3)}$ Existe muy poca información con respecto a este tipo de lesiones en la población pediátrica a nivel local así como en todo Honduras. Es por esto que el presente estudio se planteó para proporcionar un punto de partida que contribuya al mejoramiento de la casuística local para conocer los factores relacionados con este tipo de eventos, los que pueden ser prevenidos con programas de difusión. Típicamente las hospitalizaciones ocurren por lesiones graves y son costosas; conocer la magnitud de las complicaciones atribuibles a lesiones por arma de fuego entre los jóvenes caracterizaría aún más la carga de este problema de salud pública.

En el mundo hay una arma ligera por cada 10 personas y cada minuto muere una persona por un disparo. La proliferación descontrolada de armamento alimenta guerras, violaciones a los derechos humanos e impacta en la vida de millones de personas; mientras, que una minoría se beneficia de uno de los negocios más lucrativos del mundo. ${ }^{(4)}$ Las heridas por armas de fuego son una de las formas más graves de lesiones que puede sufrir el ser humano, y su severidad está relacionada no sólo con los tejidos afectados sino también con el arma utilizada. Los métodos clínicos y quirúrgicos surgidos principalmente en tiempo de guerra han llevado a una disminución progresiva de la mortalidad por dichas lesiones; sin embargo, las HPAF se han convertido en un problema importante en la vida civil.

Por ende, se hace necesario realizar la presente investigación para determinar los factores biosocioeconómicos relacionados con heridas por proyectil de arma de fuego en la población adolescente. Cabe mencionar que a nivel hospitalario local no se cuenta con estudios sobre el tema aunque el HMCR es el centro de referencia. El objetivo del estudio fue, por lo tanto, determinar los factores biológicos, sociales y económicos relacionados con heridas por proyectil disparado por arma de fuego en los pacientes adolescentes atendidos en la Emergencia de Pediatría del Hospital Mario Catarino Rivas, en el período de julio 2015 hasta junio 2017.

\section{PACIENTES Y MÉTODOS}

Es un estudio descriptivo, de tipo prospectivo, transversal, con un tipo de muestreo no probabilístico por conveniencia, de los casos de adolescentes con HPAF en el HMCR, en el período comprendido desde julio de 2015 a junio del 2017.

La población en estudio lo conformaron todos los adolescentes con diagnóstico de HPAF que ingresaron a la Emergencia de Pediatría en el período establecido siendo de 39. Se recolectaron los datos a partir de un interrogatorio que se les hizo a los familiares del paciente y al mismo con previa autorización y consentimiento informado. Se mantuvo en todo momento la confidencialidad de los datos brindados.

El instrumento se conformó por un interrogatorio el cual incluyó preguntas cerradas sobre el sexo, edad, lugar de residencia, estado civil, escolaridad, deserción escolar y su cau- 
sa, desintegración familiar, asociaciones a grupos delictivos, consumo de drogas, tabaco y/o alcohol, lugar geográfico, hora, sitio anatómico del trauma, complicaciones médicas, arma de fuego utilizada, ocupación, salario, dependientes económicos, número de habitantes en casa y actividad que realizaba al momento del trauma. Cabe mencionar que hubo una población que ni estudia ni trabaja (NiNi's) y esto va acorde a la literatura internacional. La moda de la población estudiada fue de 17 años de edad, con una media de 15.3 años.

Al momento de la revisión, se conservó la confidencialidad de los pacientes no incluyendo nombres ni datos personales y se identificaron los pacientes con sus iniciales. El análisis de datos se digitó en MS Excel.

\section{RESULTADOS}

En relación al sexo se observó que hubo predominio de hombres con $\mathrm{n}=30$ (76\%). (Ver Tabla No.1).

Tabla No. 1: Variables biológicas en adolescentes con HPAF.

\begin{tabular}{lr}
\hline & N (\%) \\
\hline SEXO & \\
Hombres & $30(76 \%)$ \\
Mujeres & $9(24 \%)$ \\
EDAD & \\
13 años & $8(20 \%)$ \\
14 años & $5(14 \%)$ \\
15 años & $7(18 \%)$ \\
16 años & $7(18 \%)$ \\
17 años & $12(30 \%)$ \\
ESTADO CIVIL & \\
Soltero & $34(87 \%)$ \\
Unión libre & $5(13 \%)$ \\
ESCOLARIDAD & \\
Primaria & \\
completa & \\
Primaria & \\
incompleta & $12(30 \%)$ \\
Secundaria & \\
NiNi's & $6(15 \%)$ \\
& $5(14 \%)$ \\
& $16(41 \%)$
\end{tabular}

\begin{tabular}{lr} 
DESERCIÓN ESCOLAR \\
$\begin{array}{l}\text { Razones } \\
\text { económicas } \\
\text { Desintegración } \\
\text { familiar }\end{array}$ \\
$\begin{array}{lr} \\
\text { LUGAR DE RESIDENCIA }\end{array}$ \\
San Pedro Sula & $6(16 \%)$ \\
Choloma & $27(69 \%)$ \\
Puerto Cortés & $4(10 \%)$ \\
Pimienta & $1(4 \%)$ \\
Potrerillos & $1(4 \%)$ \\
La Ceiba & $1(4 \%)$ \\
Tela & $2(5 \%)$ \\
\hline
\end{tabular}

Fuente: Instrumento de recolección de datos

En los adolescentes estudiados se identificó que eran de 17 años de edad $n=12(30 \%)$. El estado civil de la población estudiada fue soltero $n=34(87 \%)$.

Los adolescentes cursaron la primaria incompleta $\mathrm{n}=12$ (52\%); dentro de los mismos que abandonaron sus estudios lo hicieron por razones económicas $n=33$ (85\%). Proviniendo los mismos en gran número de San Pedro Sula n=27 (69\%).

Los factores sociales son de gran importancia en una población. (Ver tabla No. 2) Es por esto que se investigaron encontrando que los adolescentes viven en hacinamiento $n=28$ $(71 \%)$. Gran cantidad de éstos provienen de hogares desintegrados $n=23$ (58\%). Cabe mencionar que los adolescentes eran mantenidos económicamente por sus padres biológicos $n=24(61 \%)$. Laboraban $n=30(77 \%)$ y los que no estudiaban ni trabajaban $n=9$ (23\%); de la población que trabajaba devengaban un salario menor al salario mínimo $n=27(90 \%)$, siendo su propia familia la que dependía económicamente de ellos $n=26$ $(86 \%)$. Solamente $n=3(1 \%)$ de los adolescentes tuvieron asociaciones delictivas. A su vez también la mayor parte de los mismos no utilizó drogas, tabaco y/o alcohol $n=29$ (75\%). 
Las variables del traumatismo se pueden ver en la tabla 3. Encontrando que éstos ocurrieron en San Pedro Sula $n=26(67 \%)$; de éstos $\mathrm{n}=16(61 \%)$ ocurrieron en Cofradía, seguidos por Sector Chamelecón $n=4(15 \%)$ y Sector Satélite $n=4$ (15\%). Los traumatismos ocurrieron por la tarde $n=30(77 \%)$, siendo el móvil involucrado los asaltos $n=22$ (56\%). Las escuadras $(9 \mathrm{~mm})$ fueron las armas más utilizadas $n=29$ (74\%), llegando a afectar las extremidades $n=29$ (74\%); complicando médicamente a los adolescentes con fracturas $n=9(50 \%)$, seguido por hemotórax $n=7$ (39\%).

Tabla No. 2: Variables Sociales en adolescentes con HPAF.

\begin{tabular}{|c|c|}
\hline & $\mathbf{N}(\%)$ \\
\hline \multicolumn{2}{|l|}{ HACINAMIENTO } \\
\hline $\begin{array}{l}\mathrm{Si} \\
\text { No }\end{array}$ & $\begin{array}{l}28(72 \%) \\
11(28 \%)\end{array}$ \\
\hline \multicolumn{2}{|c|}{ DESINTEGRACIÓN FAMILIAR } \\
\hline $\begin{array}{l}\mathrm{Si} \\
\text { No }\end{array}$ & $\begin{array}{l}23(59 \%) \\
16(41 \%)\end{array}$ \\
\hline \multicolumn{2}{|l|}{$\begin{array}{l}\text { RESPONSABLES } \\
\text { ECONÓMICOS }\end{array}$} \\
\hline $\begin{array}{l}\text { Padres biológicos } \\
\text { Otros } \\
\text { Ninguno }\end{array}$ & $\begin{array}{r}24(61 \%) \\
7(18 \%) \\
8(21 \%)\end{array}$ \\
\hline \multicolumn{2}{|l|}{ OCUPACIÓN } \\
\hline $\begin{array}{l}\text { Trabajan } \\
\text { NiNi's }\end{array}$ & $\begin{array}{r}30(77 \%) \\
9(23 \%)\end{array}$ \\
\hline \multicolumn{2}{|l|}{ SALARIO } \\
\hline $\begin{array}{l}\text { Mínimo } \\
\text { Mayor al mínimo }\end{array}$ & $\begin{array}{r}27(93 \%) \\
2(7 \%)\end{array}$ \\
\hline \multicolumn{2}{|c|}{ DEPENDENCIA ECONÓMICA } \\
\hline $\begin{array}{l}\text { Familia } \\
\text { Ninguno }\end{array}$ & $\begin{array}{l}26(66 \%) \\
13(34 \%)\end{array}$ \\
\hline \multicolumn{2}{|c|}{ ASOCIACIÓN DELICTIVA } \\
\hline $\begin{array}{l}\mathrm{Si} \\
\text { No }\end{array}$ & $\begin{array}{r}3(8 \%) \\
36(92 \%)\end{array}$ \\
\hline \multicolumn{2}{|c|}{$\begin{array}{l}\text { USO DE DROGAS, TABACO } \\
\text { Ó ALCOHOL }\end{array}$} \\
\hline $\begin{array}{l}\text { Drogas ilegales } \\
\text { Tabaco } \\
\text { Alcohol } \\
\text { Ninguna }\end{array}$ & $\begin{array}{r}0(0 \%) \\
7(19 \%) \\
1(2 \%) \\
31(79 \%)\end{array}$ \\
\hline
\end{tabular}

Fuente: Instrumento de recolección de datos
Tabla No. 3: Variables del traumatismo en adolescentes con HPAF.

\begin{tabular}{lr}
\hline & \multicolumn{1}{c}{$\mathbf{N}(\%)$} \\
\hline LUGAR GEOGRÁFICO DE LA & \\
HPAF & \\
San Pedro Sula & $26(67 \%)$ \\
Choloma & $6(15 \%)$ \\
La Lima & $2(5 \%)$ \\
Otros & $5(13 \%)$ \\
HPAF DE S.P.S. & \\
Cofradía & $16(59 \%)$ \\
Sector Chamelecón & $4(15 \%)$ \\
Sector Satélite & $4(15 \%)$ \\
Sector Rivera Hernández & $3(11 \%)$ \\
HORA DEL TRAUMA & \\
00-06 AM & $1(3 \%)$ \\
06-12 PM & $8(20 \%)$ \\
12-06 PM & $30(77 \%)$ \\
O6-00 AM & $0(0 \%)$ \\
MÓVIL DEL TRAUMA & \\
Accidental en casa & $12(30 \%)$ \\
Accidental en vía pública & $3(8 \%)$ \\
Asalto & $22(56 \%)$ \\
Intento homicidio & $2(6 \%)$ \\
TIPO DE ARMA UTILIZADA & \\
Escuadra & $29(74 \%)$ \\
Revólver & $2(5 \%)$ \\
Fusil & $4(10 \%)$ \\
Escopeta & $1(3 \%)$ \\
Fabricación casera & $3(8 \%)$ \\
SITIO ANATÓMICO DE HPAF & \\
Extremidades & $29(60 \%)$ \\
Tórax & $10(21 \%)$ \\
Abdomen & $5(11 \%)$ \\
Glúteos & $3(6 \%)$ \\
Cráneo & $1(2 \%)$ \\
COMPLICACIONES MÉDICAS & \\
Fracturas & $9(50 \%)$ \\
Hemotórax & $7(40 \%)$ \\
Hemoperitoneo & $1(5 \%)$ \\
Craneotomía & $1(5 \%)$ \\
\hline Fention & \\
\hline
\end{tabular}

Fuente: Instrumento de recolección de datos

\section{DISCUSIÓN}

Se encontró a través del interrogatorio de 39 pacientes con diagnóstico de herida por arma 
de fuego, afecta a hombres $(76 \%)$. La relación entre sexos fue de Hombres 3.3:1 Mujeres. Estos datos son superiores a la relación mundial la cual es de 2:1 a favor de los hombres. ${ }^{(5)}$ La relación podría estar dada como consecuencia a la necesidad de laborar por lo que los adolescentes se expondría a circunstancias de riesgo. Con respecto a la edad de los pacientes; se encontró predominio de los adolescentes de 17 años de edad, representando $30 \%$ del total de los casos. Sin embargo el $50 \%$ de los casos corresponden a adolescentes entre 13 y 15 años de edad. En Honduras los adolescentes de 17 años son los más afectados por traumatismos extraños tipo heridas por arma de fuego. ${ }^{(6)}$ Estos hallazgos corresponden al hecho de que a esa edad los adolescentes salen más de sus casas y se ven obligados a trabajar para contribuir a la economía familiar.

La residencia de los pacientes estudiados fue en su gran mayoría del Departamento de Cortés con $87 \%$. De éstos el $70 \%$ residen en el municipio de San Pedro Sula y a la vez el $60 \%$ fueron de Cofradía. Éstos resultados tienen relación directa con fuentes del Instituto Nacional de Estadística (INE) en la cual la mayor parte de los casos de violencia se dan en la capital industrial Hondureña. ${ }^{(7)} \mathrm{El}$ estado civil que predominó en los pacientes afectados fue el de soltero, representando $87 \%$ de los casos. Cabe mencionar que el $13 \%$ se encontraban en unión libre y uno de ellos con hijos. Esto podría explicar la necesidad de buscar medios de sustento para sus familias, lo que ha hecho que se relacione con deserción escolar y mayor exposición a la violencia social.

En relación a la escolaridad de los pacientes estudiados, el $56 \%$ de ellos tienen primaria incompleta, esto coincide con el INE que expone que a los 15 años de edad hay un $60 \%$ de deserción escolar. ${ }^{(7)}$ Las razones del abandono de sus estudios identificadas fueron en su mayoría razones económicas, obligándolos a buscar trabajos para contribuir a la economía familiar, pero además se identificó que la desintegración familiar juega un papel importante en todo este proceso. Según el (PNUD) el año promedio de escolaridad nacional es de $4.8{ }^{\left({ }^{(8)}\right.}$

Otro factor encontrado que contribuye a la deserción escolar es el hacinamiento lo que se hace evidente en este estudio pues el $56 \%$ de los pacientes viven en esas condiciones. A nivel nacional según el INE hay $8 \%$ de la población que vive en hacinamiento. ${ }^{(9)} \mathrm{A}$ su vez hay un $2.5 \%$ de deserción escolar según la misma fuente anterior.

El $38 \%$ de los pacientes de este estudio viven en hogares disfuncionales y el $40 \%$ en hogares integrados. Según el INE, las últimas encuestas de campo realizadas hasta mayo de 2013, reflejan que de $1,898,966$ hogares al menos 1,276,056 están debidamente integrados, es decir que tienen a un hombre y una mujer como jefes de hogar; representando $33 \%$ de desintegración familiar. ${ }^{(10)}$ Siendo un factor de suma importancia dado que esto influye directamente en la necesidad de trabajar y los predispone a la deserción escolar.

El trabajo infantil es tema que concierne a muchos países a nivel mundial, siendo el mismo predominante en los países en vías de desarrollo. De los pacientes estudiados, 29 de ellos trabajaban, representando $76 \%$ del total y el $18 \%$ corresponde a pacientes que ni estudian ni trabajan. (11) Se estima según el INE que el $21 \%$ de la población juvenil de Centroamérica de entre 16 y 29 años, no estudia ni trabaja y el fondo de población de la ONU da cuenta de que el $20 \%$ de los jóvenes en el Caribe no estudia ni trabaja. Siendo casi el mismo porcentaje estimado para Centroamérica. ${ }^{(12)}$

Según estadísticas de INE, la mayor concentración del trabajo infantil se encuentra en el 
área rural con el $68.2 \%$ mientras en el área urbana se tiene el $31.8 \%$; el $74.4 \%$ son niños y el $25.6 \%$ niñas; las áreas económicas con incidencia en trabajo infantil son: la agricultura, silvicultura, caza y pesca con el $52.6 \%$; comercio por mayor y menor el $18.6 \%$; el $11.0 \%$ en la industria manufacturera, el $4.0 \%$ en construcción; transporte y almacenamiento el $1.0 \%$; explotación de minas y canteras, $0.2 \%$. ${ }^{(13)}$ La ocupación en la que la mayoría de los adolescentes estudiados labora es la de albañil con $36 \%$ del total. Viéndose también mecánicos, plomeros, pintores, carpinteros y guardias de seguridad. Encontrando que la gran mayoría de los adolescentes trabajan y los mismos devengan menos del salario mínimo $72 \%$ y dos de ellos recién un salario mayor al mínimo nacional; representando menos del $1 \%$ del total. Cabe señalar que de todos los adolescentes que trabajan, un $66 \%$ lo hace para aportar a la economía familiar, por lo cual se puede asumir y encontrar una relación al porque del abandono de los estudios al tener ellos, necesidad de mantener a su familia y ser un apoyo económico para los mismos.

También se encontraron asociaciones a grupos delictivos en el $1 \%$ específicamente asociación a maras, y todos residentes en Choloma. No existe literatura con estadística que identifica asociación de grupos delictivos con la adolescencia, pero es de importancia señalarla dado que las mismas están integradas por jóvenes entre los 13 y los 20 años de edad. ${ }^{(14)} \mathrm{De}$ todos los adolescentes estudiados ninguno ha utilizado drogas pero sí se han visto involucrados con alcohol y tabaco, representados con 3 y $18 \%$ respectivamente del total. Estudios norteamericanos demuestran estadística local de uso de alcohol y tabaco en $30 \%$ en adolescentes. ${ }^{(15)}$ Según el INE, los jóvenes a los 12 años comienzan a ingerir alcohol, los niños entre las edades de 12 a 14 años, al menos el $31 \%$ están consumiendo alcohol y el $23 \%$ fuman cigarrillos; de 15 a 17 años beben alcohol en un 55\% y fuman tabaco el $46 \%$ y de 18 a 20 años, el $70 \%$ consume alcohol y el $60 \%$ tabaco. Esto viene a demostrar que la población estudiada consumía menos alcohol y tabaco que la población nacional. ${ }^{(16)}$

En medicina, las heridas por arma de fuego constituyen una urgencia médica, llegando en algunos países a ser la principal causa de intervenciones quirúrgicas por trauma. Encontrando en los Estados Unidos que más de 10 adolescentes mueren diariamente y de ellos la mayoría se debió a heridas causadas por armas de fuego. ${ }^{(17)}$ En la actualidad en los Estados Unidos de Norteamérica se mantiene un arma de fuego en el $24 \%$ de los hogares. ${ }^{(18)}$ Esto representa casi un cuarto de todos los hogares de los Estados Unidos. En este estudio el $31 \%$ de los pacientes sufrieron HPAF a consecuencia de la manipulación del arma en casa, en Honduras a partir del 2015 se permite tener 3 armas cortas por persona según la ley del control de armas. ${ }^{(19)}$

En este estudio se encontró que el sitio anatómico afectado por la HPAF con mayor prevalencia fue las extremidades $75 \%$, siendo el segundo afectado el tórax $25 \%$. Esto concuerda con lo encontrado a nivel mundial en la cual siempre el sitio más afectado son las extremidades en $80 \%$. ${ }^{(20)}$

De todos los traumatismos el $66 \%$ ocurrió en San Pedro Sula y el segundo lugar involucrado fue Choloma con $15 \%$. En los traumas que ocurrieron en San Pedro Sula, la mayoría ocurrieron en el Sector de Cofradía, seguido por Chamelecón, Satélite y Rivera Hernández respectivamente. Cabe señalar que éstas áreas de la ciudad de San Pedro Sula, son consideradas áreas de conflicto social. ${ }^{(21)}$ Se identificó que el arma más comúnmente utilizada fue la escuadra en el $75 \%$ de los casos, la segunda utilizada fueron los fusiles con 10\%. Esto no concuerda con las estadísticas del INE que reporta 
que el arma las comúnmente utilizada son las tipo revolver. ${ }^{(22)}$ Las armas de fabricación casera también fueron responsables de 3 traumatismos $8 \%$. A nivel nacional ha habido un repunte de distribución de armas caseras, siendo estas consideradas al nivel de las fabricadas internacionalmente. ${ }^{(23)}$

El horario que predominó en los casos de los traumatismos fue por la tarde con $76 \%$ de los casos, seguidos por la mañana $20 \%$. Estadísticas del INE revelan que en Honduras, en uno de cada tres hogares del país vive un hondureño que ha sido víctima de un asalto. De todos éstos la mayoría ocurren de día y tardan un promedio de 2-3 minutos. Datos que concuerdan con la hora del día predominante de los traumatismos en la población estudiada y se encontró que la mayoría fueron los asaltos con $56 \%$. De todos los casos estudiados, el $46 \%$ tuvo complicaciones médicas. Las observadas fueron hemotórax, hemoperitoneo, craneotomía y fracturas siendo esta última la más frecuente en $23 \%$ de los pacientes. Según la OMS, los principales sitios afectados por las HPAF son en abdomen, seguido por las extremidades y luego el tórax. ${ }^{(24)}$ La principal área anatómica afectada por HPAF en los pacientes fueron las extremidades. En estudios internacionales se ven complicaciones mayores, incluyendo la muerte, lo cual afortunadamente no ocurrió en ninguno de los pacientes de este estudio. Por ende se recomienda que es necesario realizar más estudios dirigidos a detectar los principales factores de riesgo, para entonces diseñar estrategias preventivas adecuadas en coordinación con las autoridades y la comunidad.

Es por esto que se recomienda que: El Estado debe promover el desarme general, limitar la venta de municiones, controlar la compra de armas por medio de un examen psicométrico para la población y la obligatoriedad del certificado de portación de armas.

La Secretaría de Salud debería de concientizar a la población de los peligros asociados con las armas de fuego, sobre todo en la población pediátrica. El registro continuo de estos casos con el fin de identificar la casuística y diseñar estrategias de prevención. La población hondureña debe de guardar las armas de fuego, sin balas y desmartilladas, en un envase cerrado con llave. Sólo los padres deben de saber dónde se guarda el envase. Guardar las balas por separado de las armas y también bajo llave.

Para los revólveres, colocar un candado alrededor de la barra superior para prevenir que pueda cerrarse el cilindro, o use un candado de gatillo; para las escuadras, use un candado de gatillo. Al limpiar un arma de fuego o al tenerla en sus manos por cualquier razón, nunca la deje desatendida ni por un segundo; debe mantenerla a la vista en todo momento. 


\section{REFERENCIAS BIBLIOGRÁFICAS}

1. Carter, P. M., Walton, M. A., Newton, M. F., Clery, M., Whiteside, L. K., Zimmerman, M. A., \& Cunningham, R. M. Firearm Possession Among Adolescents Presenting to an Urban Emergency Department for Assault. [Internet] Pediatrics, 132 (2), 213-221. http://doi.org/10.1542/peds.2013-0163 [Citado el 10 de abril de 2017].

Disponible en: www.pediatrics.com.

2. Comisión de Jefes, y Directores de Policía de Centroamérica, México y el Caribe. Armas de Fuego en Centroamérica. DELINCUENCIA ORGANIZADA TRANSNACIONAL EN CENTROAMÉRICA Y EL CARIBE. 2010: p. 49. [INTERNET] Senado Mexicano 2014 [Citado el 10 de abril de 2017]

Disponible en: www.senado.gob.mx.

3. Secretaría de Estado en el Despacho de Seguridad. LEY DE CONTROL DE ARMAS DE FUEGO, MUNICIONES EXPLOSIONES Y OTROS SIMILARES. DIARIO OFICIAL LA GACETA. [INTERNET] 2014 [Citado el 10 de abril de 2017] Disponible en: www.tsc.gob.hn.

4. Castellanos J. Honduras: La violencia en cifras. Tegucigalpa: Instituto Nacional de Estadísticas. [INTERNET] 2013. [Citado el 10 de abril de 2017]

Disponible en: www.ine.gob.hn.

5. Leventhal J, Gaither J, Sege R. Hospitalizations Due to Firearm Injuries in Children and Adolescents. [INTERNET] PEDIATRICS. 2014 [Citado el 10 de abril de 2017]. Disponible en: www.pediatrics.com.

6. Sotelo Cruz N, Cordero Olivares A, Woller Vásquez R. Heridas por proyectil de arma de fuego en niños y adolescentes. [INTERNET] Cirugía y Cirujanos. 2014. [Citado el 10 de abril de 2017].
Disponible en: www.nejm.com.

7. Martínez Saleh LM, De la O Cavazos ME, Elizondo Omaña RE, Guzmán López S, Valenzuela Montoya JC. Prevalencia de lesiones por arma de fuego en pacientes pediátricos tratados en el servicio de urgencias de pediatría del Hospital Universitario Dr. José Eleuterio González. Salud Pública de México. [INTERNET] 2013 [Citado el 10 de abril de 2017] Disponible en: www.senado.gob.mx.

8. Teplin L, Jakubowski J, Abram K, Olson $\mathrm{N}$, Stokes M, Welty L. Firearm Homicide and Other Causes of Death in Delinquents: A 16-Year Prospective Study. [INTERNET] PEDIATRICS. 2015 [Citado el 10 de abril de 2017].

Disponible en www.pediatrics.com.

9. Romer D, Jamieson $P$, Bushman B, Bleakley $A$, Wang $A$, Langleben $D$, et al. Parental Desensitization to Violence and Sex in Movies. [INTERNET] PEDIATRICS. 2014 [Citado el 10 de abril de 2017]. Disponible en: www.pediatrics.com.

10. Schaechter J. Prevent Youth Assault by Assaulting Firearm Violence. [INTERNET] PEDIATRICS. 2015. [Citado el 10 de abril de 2017].

Disponible en www.pediatrics.com.

11. Newgard C, Kupperman N, Holmes J, Haukoos J, Brian W, Hsia R, et al. Gunshot Injuries in Children Served by Emergency Services. [INTERNET] PEDIATRICS. 2013 [Citado el 10 de abril de 2017]. Disponible en www.pediatrics.com.

12. Nance M, Oldham K, Krummel T. Firear$\mathrm{ms}$, Children, and Health Care Professionals. [INTERNET] PEDIATRICS. 2014 [Citado el 10 de abril de 2017]. Disponible en www.pediatrics.com. 
13. Carter $P$, Walton M, Roehler D, Goldstick J, Zimmerman M, Blow F, et al. Firearm Violence Among High-Risk Emergency Department Youth After an Assault Injury. [INTERNET] PEDIATRICS. 2015 [Citado el 10 de abril de 2017].

Disponible en www.pediatrics.com.

14. Pickett W, Craig W, Harel Y, Cunningham J, Simpson K, Molcho M, et al. Cross-national Study of Fighting and Weapon Carrying as Determinants of Adolescent Injury. [INTERNET] PEDIATRICS. 2016 [Citado el 10 de abril de 2017].

Disponible en www.pediatrics.com.

15. Council on Injury, Violence and Poison Prevention Executive Committee. Firearm-Related Injuries Affecting the Pediatric Population. [INTERNET] PEDIATRICS. 2012 [Citado el 10 de abril de 2017].

Disponible en www.pediatrics.com.

16. Study: 20,000 children each year sent to EDs with firearm injuries Melissa Jenco et al.. [INTERNET] AAP News. 2016 [Citado el 10 de abril de 2017].

Disponible en www.aappublications.org.

17. Study: Gunfire kills or injures more than 7,000 children per year Melissa Jenco et al. [INTERNET] AAP News. 2015 [Citado el 10 de abril de 2017].

Disponible en www.aappublications.org.

18 Children's National Health System. (2017, May 5). Regions with stronger gun laws have fewer gun-related pediatric emergency department visits: Five-year national study could support changes in regional firearm legislation. [INTERNET] AAP News. 2016 [Citado el 10 de abril de 2017].

Disponible en www.aappublications.org.

19. Washington University in St. Louis.
(2016, September 14). Half of kids in American families studied spend time in households with firearms. [INTERNET] AAP News. 2016 [Citado el 10 de abril de 2017].

Disponible en www.aappublications.org.

20. American Academy of Pediatrics. (2017, May 4). Youth most at risk for violence or mental health issues have increased access to guns: Teens with a past suicide attempt or mental health disorder also have increased access to firearms -either in their own home or a friend's. [INTERNET] AAP News. 2016 [Citado el 10 de abril de 2017].

Disponible en www.aappublications.org.

21. Fowler KA, Dahlberg LL, Haileyesus $T$, Annest JL. Firearm injuries in the United States. [INTERNET] PEDIATRICS. 2015. [Citado el 10 de abril de 2017].

Disponible en www.pediatrics.com.

22. Fowler KA, Dahlberg LL, Haileyesus T, et al. Childhood Firearm Injuries in the United States. [INTERNET] PEDIATRICS. 2017. [Citado el 10 de abril de 2017].

Disponible en www.pediatrics.com.

23. Richardson EG, Hemenway D. Homicide, suicide, and unintentional firearm fatality: comparing the United States with other high-income countries, [INTERNET] PEDIATRICS. 2015. [Citado el 10 de abril de 2017].

Disponible en www.pediatrics.com.

24. Schaechter J, Duran I, De Marchena J, et al. Are "accidental" gun deaths as rare as they seem? A comparison of medical examiner manner of death coding with an intent-based classification approach. [INTERNET] PEDIATRICS. 2015. [Citado el 10 de abril de 2017].

Disponible en www.pediatrics.com. 